\title{
HIGH FIELD-GRADIENT CAVITIES LOADED WITH MAGNETIC ALLOYS FOR SYNCHROTRONS
}

\author{
C. Ohmori*, E. Ezura, M. Fujieda, Y. Mori, R. Muramatsu, H. Nakayama, \\ Y. Sato, A. Takagi, M. Toda, T. Uesugi, M. Yamamoto, and M. Yoshii, KEK \\ M. Kanazawa and K. Noda, NIRS
}

\section{Abstract}

Very high field-gradient has become available by a new Magnetic Alloy (MA)-loaded cavity developed for high intensity proton synchrotrons. The available RF voltage per core is ten times larger than that of the ordinary ferrite core. The maximum voltage of $20 \mathrm{kV}$ has been achieved by the High-Field Gradient Cavity(HGC) of $40 \mathrm{~cm}$ in length[1].

Because the intrinsic Q-value of the MA core is low, acceleration without any tuning system also becomes possible. The first beam acceleration test using the HGC has been performed successfully at the HIMAC(Heavy Ion Medical Accelerator in Chiba). Furthermore, the dual harmonic RF and barrier bucket experiments have been carried out.

Another advantage of the MA-loaded cavity is that it is easy to compensate the beam loading. The feed forward beam compensation was applied for both HGC on the test bench using electron beam and MA-loaded cavity installed in the AGS for the barrier bucket experiment. A new development for high-Q HGC using cut core configuration will be also reported.

\section{INTRODUCTION}

The field gradient of a RF cavity is one of the most important parameters to design a high intensity machine. In order to minimize the circumference of the synchrotron and to increase the repetition rate of acceleration cycle for the high beam current, high field gradient is required. In case of JHF(Japan Hadron Facility)[2], the total RF voltage of $420 \mathrm{kV}$ is necessary for the $3 \mathrm{GeV}$ Booster synchrotron. Some future upgrades including the energy up to 5-6 GeV and/or $50 \mathrm{~Hz}$ operation require about $800 \mathrm{kV}$. So far, the maximum field gradient in the frequency region of 2-3.4 $\mathrm{MHz}$ was about $15 \mathrm{kV} / \mathrm{m}$ for the ferrite-loaded cavity because of the characteristics of the ferrite core. The shunt impedance of ferrite core becomes very low at the magnetic flux density of few 100 Gauss because the saturation flux density(Bs) is small as shown in Fig. 1. If ordinary ferrite-loaded cavities are used for the JHF, the total length for them becomes about $60 \mathrm{~m}$. It means that most of the long straight sections are occupied for the RF stations.

To achieve high field gradient, the characteristics of the magnetic core should be stable in high RF field. Recently, soft magnetic alloys (MA) with high permeability, such as FINEMET[3], Metglas[4] and other amorphous have been developed. Some of the cores have very high saturation

\footnotetext{
*E-mail: chihiro@insuty.tanashi.kek.jp
}

flux density of more than $10 \mathrm{kGauss,}$ as shown in Fig. 1. On the other hand, the hysteresis loss in the ferrite core will be increased at the large RF amplitude. However, the loss in the MA core does not depend on the RF amplitude. The MA has conductivity and the RF power is lost mainly by the eddy current in the material. To reduce the eddy loss, the material is composed of the tape of $16 \mu \mathrm{m}$ thickness which is insulated by a thin Silica layer.

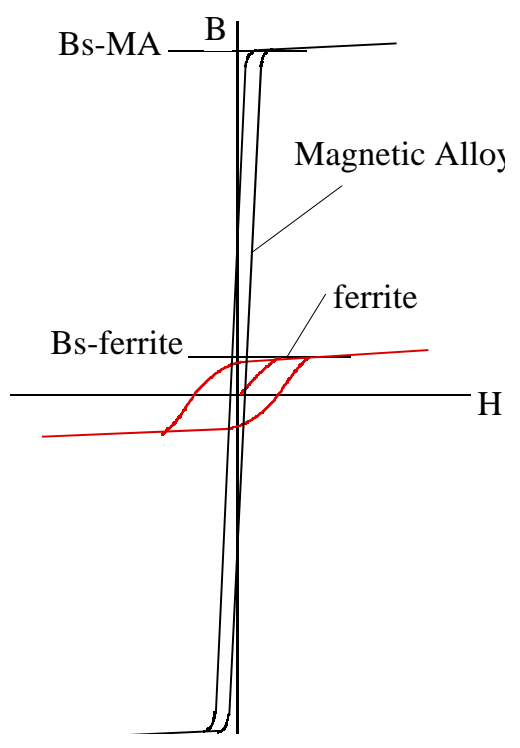

Figure 1: The hysteresis curves of magnetic materials. $B_{s}$ means the saturation magnetic flux density. The usable $B_{R F}$ is much smaller than $B_{s}$.

The characteristics of the MA and ferrite cores have been measured by a test bench using a $1 \mathrm{~kW}$ transistor amplifier[3]. The results are shown in Fig. 2. To evaluate the characteristics of magnetic materials, the product of relative permeability $(\mu)$, quality factor of the material $(\mathrm{Q})$ and frequency(f), so called $\mu Q f$-product has been used. The $\mu Q f$-product is given by $R p=\mu_{0} \times t \times \ln (a / b) \times(\mu Q f)$, where $R_{p}$, t, a and $\mathrm{b}$ are shunt impedance, thickness, outer diameter and inner diameter, respectively. The $\mu Q f$ product depends on the magnetic flux density in a core, $B_{R F}$. It is given by $B_{R F}=\frac{V}{\omega S}$, where $\mathrm{V}$ and $\mathrm{S}$ are the voltage and cross section of the core, respectively. $B_{R F}$ is the averaged value in the core and the flux density near the inside is larger than it. The MA core shows an excellent characteristics at very high magnetic flux density of 2 kGauss. The characteristics of a ferrite core are also 
shown in the figure. Below 100 Gauss, ferrite core has larger $\mu Q f$-product than the MA core. However, the MA has larger value at the large RF amplitude. Another measurement using $30 \mathrm{~kW}$ tetrode amplifier also shows a large MA core of $550 \mathrm{~mm}$ O.D. was stable at the magnetic flux density of $1.3 \mathrm{kGauss}$ and $5.5 \mathrm{kV}$ was obtained by the core which had the thickness of $2.5 \mathrm{~cm}$.

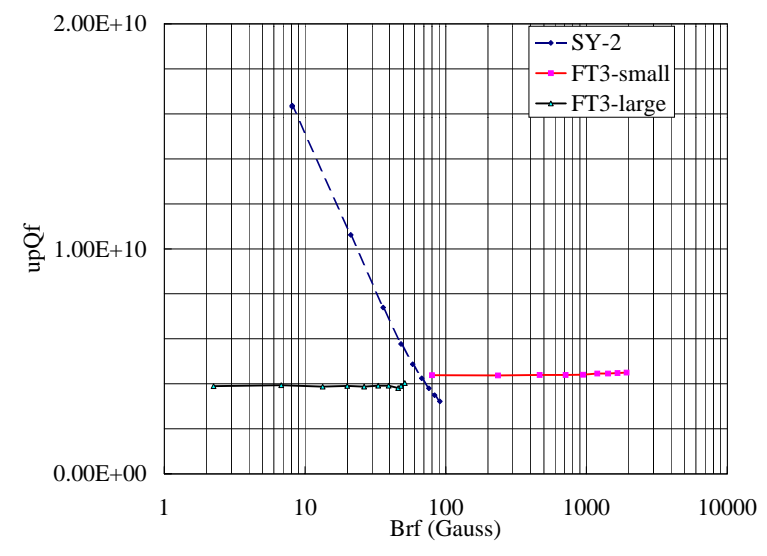

Figure 2: Magnetic flux density dependence of magnetic materials. SY2 is a typical ferrite core and the magnetic flux density dependence was observed. FT3-small and large are MA cores of 570mm O. D. and $70 \mathrm{~mm}$ O.D., respectively. The MA cores have a constant shunt impedance up to $2 \mathrm{kGauss}$.

The characteristics of MA core are summarized as followings;

(1) $\mu Q f$-product of the MA core does not depend on the flux density. One of MA cores, FINEMET, has larger saturation flux density than other MA and can be used at the very high RF flux density of 2 kGauss.

(2) The intrinsic Q-value of the MA core is 0.6-1. It is suitable for the wide frequency sweep without tuning system, barrier bucket, and multi-harmonic RF acceleration.

(3) The permeability of the MA core is about 10 times higher than that of ferrite. Although the Q-value is low, the $\mu Q f$-product is not small to use for RF cavity.

(4) The MA core has very high Curie temperature of $570^{\circ} \mathrm{C}$. And the characteristics of the core remained constant at high temperature of $170^{\circ} \mathrm{C}$.

(5)The Q-value of the core can be changed up to more than 10 by using the cut core technique(see section 5). The $\mathrm{R} / \mathrm{Q}$-value of the cavity is a variable without changing the shunt impedance.

(6) The core consists of thin MA tape which is coated by silica insulator. It is possible to make a large core of about $100 \mathrm{~cm}$ diameter.

\section{HIGH-FIELD GRADIENT CAVITY(HGC)}

\subsection{High Field Gradient}

A prototype of HGC has been developed as shown in Fig. 3 . The cavity has one acceleration gap and consists of two sets of $\frac{1}{4} \lambda$ cell. Each cell composed of 3 MA cores and they are installed in a water tank to cool by water directly. The size of MA cores is $305 \mathrm{~mm}$ I.D., 670mm O.D. and $26 \mathrm{~mm}$ thickness. The impedance of the cavity is shown in Fig. 4. The impedance is very broad because of the characteristics of core. The cavity has been driven by a push-pull amplifier using two sets of $150 \mathrm{~kW}$ tetrode, 4CW150,000. The maximum voltage of $20 \mathrm{kV}$ has been achieved for the pulse operation. The field gradient per cavity length was $50 \mathrm{kV} / \mathrm{m}$. For the high duty operation, $15 \mathrm{kV}$ has been obtained. The voltage and duty are limited by the capacities of the plate power supply and cooling water.

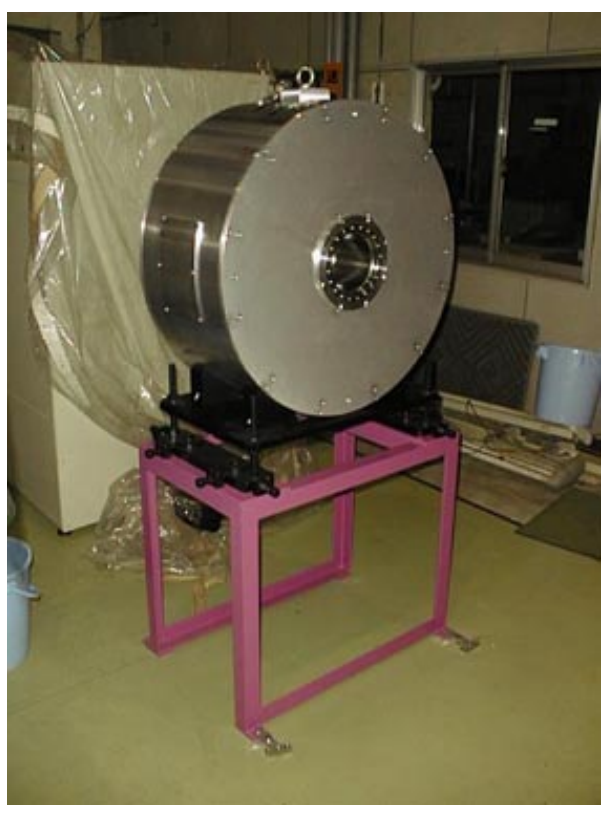

Figure 3: High field gradient cavity. The length of the cavity is $40 \mathrm{~cm}$.

\subsection{Fast Frequency Sweep}

Because of the broadband impedance of the MA core, any delay effcts on the magnetic material, such as a magneticafter effect of ferrite core, has not been observed. Very fast repetition of about $1 \mathrm{kHz}$ for the frequency sweep has been achieved. Figure 5 shows the envelope of the gap voltage when the 1-5 MHz frequency sweep was performed in 1.1 ms.

\section{BEAM TEST AT HIMAC}

The HGC has been installed in the HIMAC for the beam test. The HIMAC is a heavy ion synchrotron for the cancer 


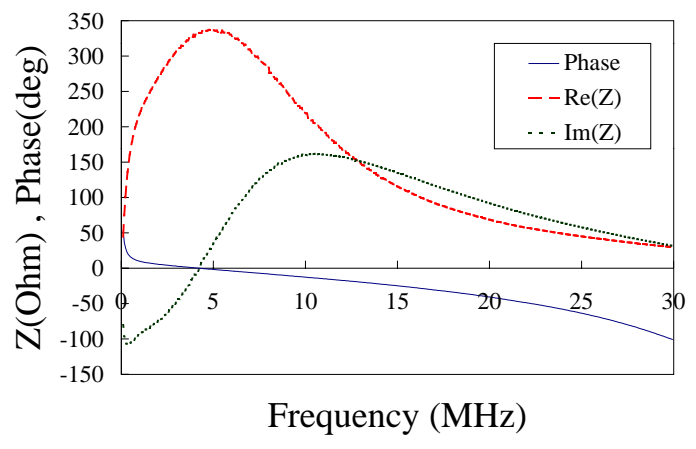

Figure 4: Very broad impedance curve of the HGC. The resonance frequency was shifted when the high power amplifier was connected.

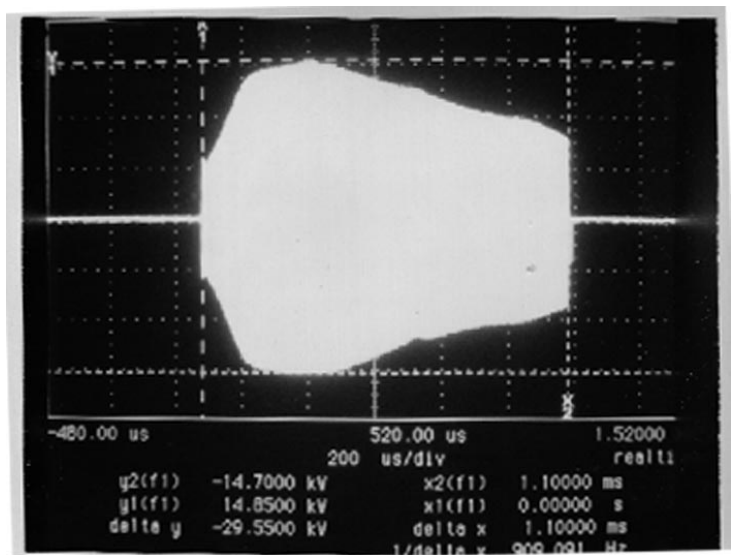

Figure 5: The envelope of the gap voltage while the fast frequency sweep was performed. Because of the characteristics of the drive amplifier, the RF voltages was low at 1 $\mathrm{MHz}$.

therapy. The main parameters of the HIMAC are listed in Table 1.

Table 1: Main Parameters of HIMAC

\begin{tabular}{|l|c|}
\hline Circumference & $129.6 \mathrm{~m}$ \\
Injection Energy & $6 \mathrm{MeV} / \mathrm{u}$ \\
Extraction Energy & $800 \mathrm{MeV} / \mathrm{u}$ \\
RF frequency & $1-8 \mathrm{MHz}$ for Si \\
& $1-5 \mathrm{MHz}$ for He \\
Harmonic Number & 4 \\
Repetition & $0.3 \mathrm{~Hz}$ \\
Ramping Time & $1 \mathrm{~s}$ \\
Beam Intensity & $1.4 \times 10^{11}$ at Injection \\
Extraction & Slow Extraction \\
\hline
\end{tabular}

\subsection{Beam Acceleration without Any Feedback Loop}

Beam acceleration test has been performed for several ion beams, $\mathrm{He}, \mathrm{C}, \mathrm{Ne}$ and $\mathrm{Si}[5]$. Because the HGC has a very broad impedance, it can be used for 1-8 MHz without the tuning system. The block diagram of the HGC accelerating system is shown in Fig. 6. The preprogrammed voltage control system was employed to obtain the constant RF voltage during acceleration. Without preprogramming, the $\mathrm{RF}$ voltage became rather low at the particular frequencies because of the gain characteristics of the driver amplifier. The RF voltage was gradually changed during the adiabatic capture process and beginning of the acceleration. The preprogramming controlled the gap voltage accurate enough to accelerate the beam without beam loss. The AGC(Auto Gain Control) loop was not necessary to control the gap voltage. The RF frequency pattern for the beam acceleration using another ferrite-loaded cavity is stored in the memory of a DDS system. The same RF frequency pattern was used for the beam acceleration using the HGC.

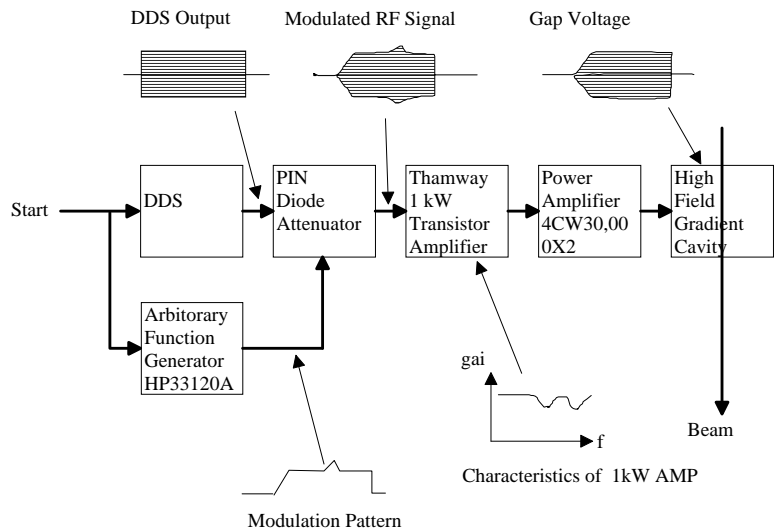

Figure 6: The Block Diagram of the HGC accelerating system.

\subsection{Multi-harmonic Experiments}

The HGC RF system is broadband and can generate the multi-harmonic frequencies on the gap simultaneously[6]. The dual harmonic RF was applied successfully. The fundamental RF frequency is divided by a splitter and doubled by a frequency doubler circuit. Both harmonic frequencies were combined after adjusting the phase by a phase shifter circuit. The amplitudes of both frequencies were controlled by PIN diode attenuators. The bunching factor of the beam was increased from 0.28 to 0.40 by the dual harmonic RF as shown in Fig. 7. The beam loss was reduced. The DDS system for the multi-harmonic RF is under development for the dual harmonic acceleration. Another multi-harmonic experiment to produce the saw-tooth RF on the gap has been performed[6]. 


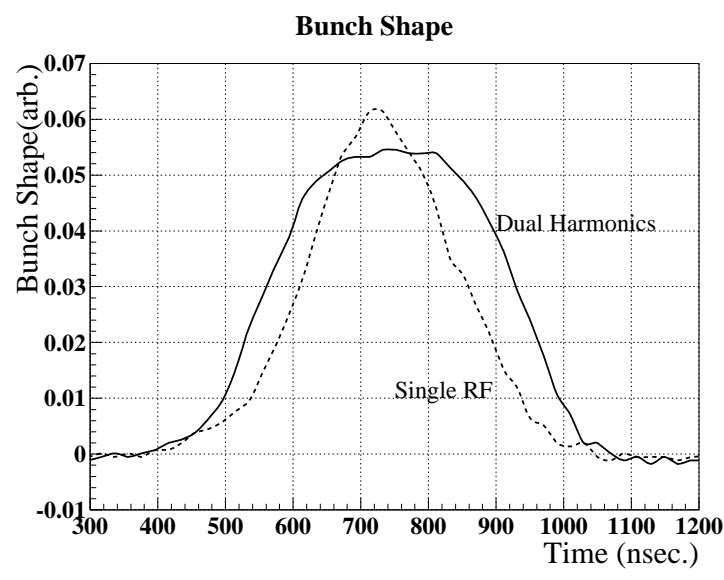

Figure 7: The difference of the bunch shapes by applying the dual harmonics.

\subsection{Bunch Compression By Barrier Voltages}

The barrier RF experiment has been carried out for the bunch compression experiment[6]. The wideband RF system is suitable for the barrier bucket experiment[7, 8]. Two barrier RF voltages were excited by the single HGC. The shape of the barrier is under development. Figure 8 shows the gap voltage after the improvement. The overshoot has been disappeared.

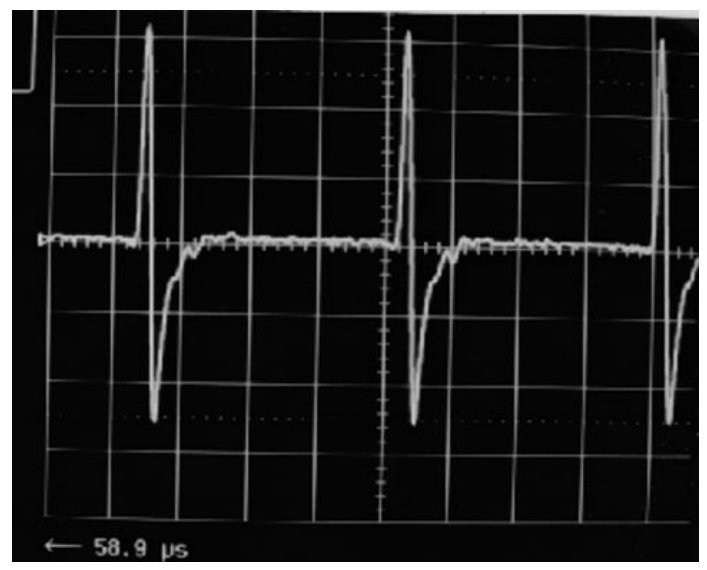

Figure 8: The barrier RF voltage. The barrier voltage is 1.1 $\mathrm{kV}$ and the repetition of the barrier RF is $261 \mathrm{kHz}$.

\section{BEAM LOADING}

\subsection{Beam Loading Compensation Test using Electron Beam}

The beam loading is one of the most severe problems on the RF system for the high intensity accelerator. The HGC has low impedance and this is suitable for the stational beam loading because the relative loading parameter becomes small. However, R/Q-value is not ideal because of low
Q-value. The beam loading effects on the HGC has been tested by the electron beam[10]. The energy of the electron beam is $180 \mathrm{keV}$ and it is modulated with the frequency of $3 \mathrm{MHz}$ to simulate the circulating beam. The length of the pulse train is $5 \mu \mathrm{sec}$. The electron beam current was measured by a fast current transformer and faraday cup. The beam transmission rate was almost $90 \%$. The wake voltage was about $1.2 \mathrm{kV}$ when the electron beam peak current was $8.6 \mathrm{~A}$.

The wake voltage was compensated by the feed forward scheme as shown in Fig. 9. The electron beam current picked up by the CT was divided into 3 harmonics $(3,6,9$ $\mathrm{MHz}$ ) by the filters, the gain and delay were adjusted, individually, and all harmonics were combined together. The combined signal was returned to the driver amplifier. These 3 harmonics were compensated, remarkably[11].

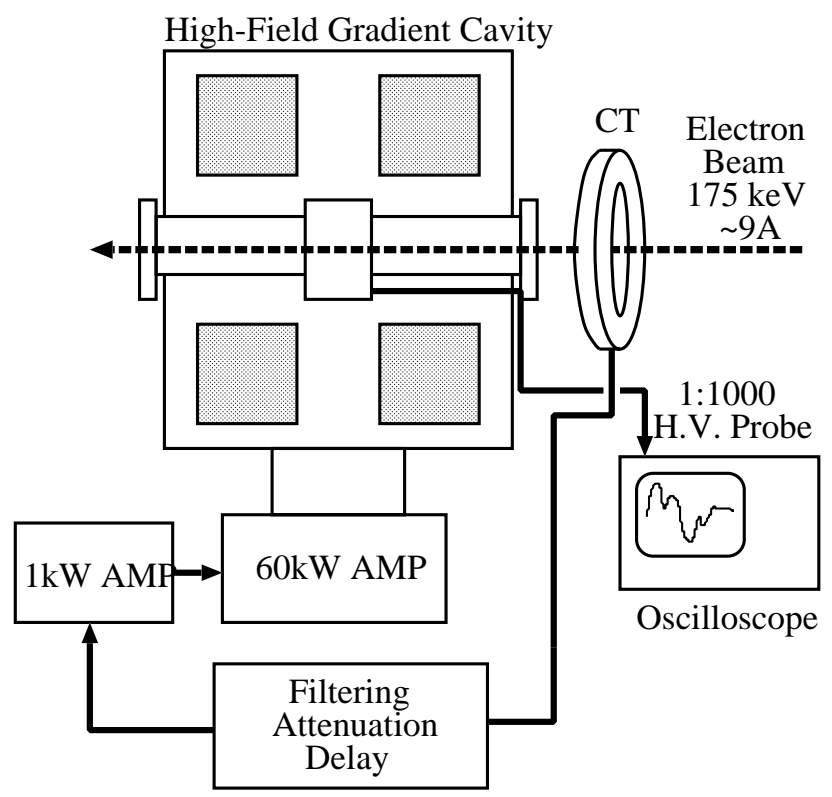

Figure 9: Set up of the compensation test system.

\subsection{Feed Forward Beam Compensation at AGS}

A MA-loaded cavity has been installed in the AGS for the barrier bucket experiments[8]. The feed forward technique was applied to compensate it and the voltage induced by the $8 \times 10^{12}$ proton per bunch was reduced up to $1 / 9$ successfully[7].

\section{CORE IMPROVEMENTS}

\subsection{Cut Core}

The Q-value of the MA core can be increased by use of a radial gap in the cut core configuration without having large deterioration of the $\mu Q f$-product. Figure 10 shows the Qvalue of the MA core for the various radial gap height. By this improvement, R/Q-value of the cavity will be reduced remarkably. In the JHF-50 GeV main ring, the peak beam 
current reaches more than 100 A because the bunching factor will be 0.05 at the top energy. The very low R/Q-value less than $100 \Omega$ is suitable to handle the bucket distortion by such a high peak beam current. However, we should note that the beam loading for low-Q system can be easily compensated. Furthermore, it is pointed out that Q-value should avoid around $\mathrm{H} / 2$ where $\mathrm{H}$ is the harmonic number of the ring to reduce the transient beam loading effects [9]. There are two choices for the JHF RF, that is, high-Q HGC which is very strong for the beam loading and low-Q HGC which is suitable for beam gymnastics and for beam loading compensation.

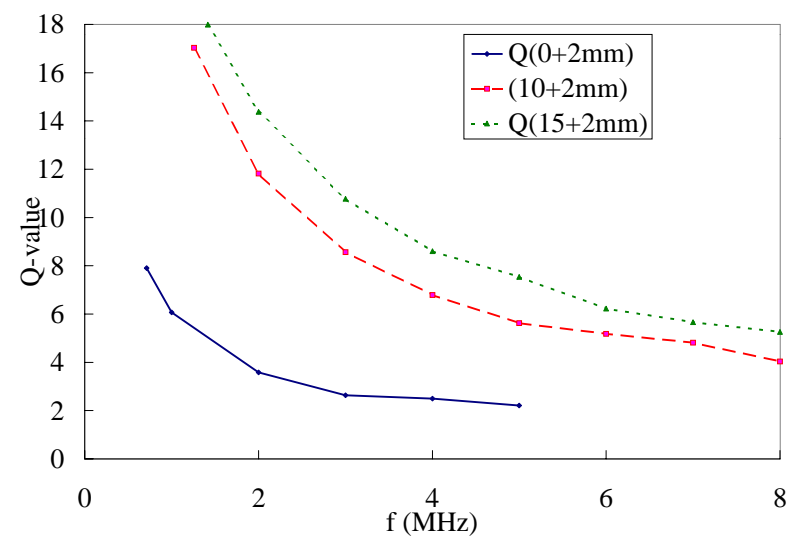

Figure 10: The Q-value of the cut core vs. gap height.

\subsection{Variable-QHGC}

In order to investigate the optimum Q-value of the HGC, a variable-Q HGC is now under development. For the cavity, large cut cores of $950 \mathrm{~cm}$ O.D. will be used. The cavity has two gaps and will be driven by a new high power amplifier which use two set of $600 \mathrm{~kW}$ tetrode, TH558. The beam loading effects including the transient process will be examined by the electron beam test bench. For the experiments, a new electron gun will be used to obtain the peak current of $30 \mathrm{~A}$.

\section{CONCLUSION}

High-Field Gradient Cavity has been developed using a newly developed Magnetic Alloy and the field gradient of $50 \mathrm{kV} / \mathrm{m}$ becomes possible. In the core, the field gradient of $220 \mathrm{kV} / \mathrm{m}$ has been achieved. Q-value of the cavity can be changed from 0.6(intrinsic Q-value) to more than 10 by the cut core configuration. A low-Q HGC is suitable for the barrier bucket scheme, multi-harmonic acceleration and wide frequency sweep. It is also easy to compensate the beam loading on the cavity using the feed forward technique. The scheme was applied for the HGC at the electron beam test bench and for a MA-loaded cavity installed in the AGS for barrier bucket experiment. The voltage by the intense AGS beam becomes $1 / 9$ by this method. High-
Q HGC is now under development and it will be suitable for high intensity accelerators because of its low R/Q-value and small shunt impedance.

\section{ACKNOWLEDGEMENT}

We would like to thank operating crew of Accelerator Engineering Corporation for operation of HIMAC facility. We are grateful to Drs. Y. Tanabe, K. Saito, T. Yan, K. Koba, A. Noda, Y. Iwashita, K. Sato, F. Pedersen, R. Garoby, M. Meth, T. Roser and M. Brennan for their advices and comments for the HGC and MA.

\section{REFERENCES}

[1] Y. Mori et al., "A New Type of RF Cavity for High Intensity Proton Synchrotron Using High Permeability Magnetic Alloy", Proc. of EPAC98, p299-301.

[2] Y. Yamazaki et al., in this conference.

[3] T. Uesugi et al., "New magnetic material for proton synchrotron rf cavity", Proc. of Symp. on Acc. Sci. and Tech., Nishiharima, Japan 1997.

[4] I. Bolotin et al., "Ferromagnetic Cores Made from Amorphous Material for Broad-band accelerating System" Proc. of PAC95, p1833-1835.

[5] R. Muramatsu et al., in this conference.

[6] M. Yamamoto et al., in this conference.

[7] M. Fujieda et al., in this conference.

[8] M. Fujieda et al., "MA-Loaded Cavity for Barrier Bucket Experiment", Proc. of EPAC98, p1796-1798.

[9] M. Yamamoto et al., "Beam Loading Effects in JHF Synchrotrons", Proc. of APAC98, p399-402.

[10] Y. Hashimoto et al., "Beam Loading Experiment with Short Bunched Electron Beam for New Type of Accelerating RF System of High Intensity Proton Synchrotron", Proc. of EPAC98, p1770-1772.

[11] M. Yamamoto et al., in this conference. 